\title{
Green Synthesis of Silver Nanoparticles using Bark Extract of Salix Alba and its Antimicrobial Effect Against Bacteria Isolated from Dental Plaque
}

\author{
SHAHNAZ MAJEED ${ }^{1 *}$ and MUSSARAT KHANDAY ${ }^{2}$ \\ ${ }^{1}$ Faculty of Pharmacy and Health science Universiti of Kuala lumpur, \\ Royal College of Medicine, Ipoh Perak, Malaysia. \\ ${ }^{2}$ Department of Microbiology Annamalai University, Chidambaram, Tamil Nadu India. \\ ${ }^{*}$ Corresponding author E-mail : shahnazmajeed5 @ gmail.com \\ http://dx.doi.org/10.13005/ojc/320337
}

(Received: April 13, 2016; Accepted: May 23, 2016)

\begin{abstract}
Now a days oral infections are major concern for the researchers due to chewing large amount of Tabaco, poor hygiene of mouth as well as smoking. These common life styles can enhance the oral infection as well as can play an important role in enhancing the bacterial resistance by the accumulation of antibiotics. Hence my present work was to focused on the biosynthesis of silver nanoparticles using Salix alba bark extract. The color of the plant extract changes in to dark brown upon addition of silver nitrate indicates the formation of silver nanoparticles which completes the reaction after 24 hours.. These nanoparticles were characterized by different microscopic techniques like UV- Vis spectrophotometry which showed the absorption peak at $440 \mathrm{~nm}$ specific for silver nanoparticles. HR-TEM showed average size was between $29-35 \mathrm{~nm}$ and the tiny particles were seemed to be spherical in morphology. Zeta potential showed silver nanoparticles were stable due to the electrostatic repulsion without adding a different physical or chemical capping agent. Atomic Force Microscopy (AFM) showed nanoparticles size was $30-50 \mathrm{~nm}$ and were stable. . The dental caries forming bacteria were isolated from plaque samples like Lactobacillus $\mathrm{sp}$, Streptococcus $s p$. and Staphylococcus sp. and compare with different reference strains. Antibacterial activity of these biologically synthesized silver nanoparticles was evaluated by disc diffusion. These synthesized silver nanoparticles showed a good antibacterial activity against the bacteria isolates. . Furthermore these nanoparticles showed quite significant activity along with different antibiotics like Amoxicillin, Tetracycline and Ciprofloxacin during the present study.
\end{abstract}

Keywords: Salix alba, AFM, HRTEM, UV- vis spectrophotometer, Zeta- potential , antibacterial effect.

\section{INTRODUCTION}

In the present century the population of microbes are getting resistant to antibiotics are increasing continuously due to the use of large amount of antibiotics, and their control is a major challenge for researchers and pharmaceutical companies ${ }^{1}$ to develop an alternative source which 
counter the bacterial resistance. Silver and silver nanoparticles are known from ancient times contain good antibacterial. Silver nanoparticles have wide range of application used as antimicrobial coatings, textiles, wound dressing, biomedical devices and can be used in refrigerators as well as in washing machines as an antibacterial material.. Silver nanoparticles shows very good antibacterial activity against gram positive as well as gram negative bacteria including multi-resistant strains ${ }^{2}$, and also it was found to be in few studies ${ }^{3}$.

Silver nanoparticles can synthesized by physical, chemical and biological method, but using biological method especially various plants and their extracts can be advantageous over other biological synthesis processes which involve the very complex procedures of maintaining microbial cultures ${ }^{4}$. The biosynthesis of silver nanoparticles using plant extracts is the most adopted method as it is eco-friendly, cheap and easily available.Plants used as a source for the synthesis of nanoparticles has a special advantage that the plants are widely distributed, much safer to handle easily obtainable, and act as a source of several metabolites ${ }^{5}$.

In the recent days, silver nanoparticles have been synthesized from the naturally occurring sources and their products like leguminous shrub (Sesbania drummondii), green tea (Camellia sinensis), Neem (Azadirachta indica) , , various leaf broths, starch, Aloe vera plant extract, lemongrass leaves extract, etc ${ }^{6}$. However for the past few years, chemical methods for the synthesis of nanoparticles have been replaced by green synthesis because the concern has been raised on the toxicity of chemical agents used for the synthesis. Thus, it is essential to develop a green approach for AgNPs production without using hazardous substances to the human health and environment. Compared with the traditional synthetic methods, biological systems provide a novel idea for the production of nanomaterials? ${ }^{7}$.

In present investigation we have reported the biosynthesis of silver nanoparticles using bark extract of salix alba. These nanoparticles were further characterized by various microscopic techniques their antibacterial effect against bacteria isolated from dental plaque.

\section{MATERIAL AND METHODS}

\section{Collection of dental Plaque sample}

The samples were taken from 10 patients with visible caries formation, attending the outdoor patients department of the Rajah Muthiah Dental College and Hospital Annamalai University. A written permission was taken from the authorities for collection of samples. A small scraping from the dental plaque was taken using a sterile forceps. The plaques were transferred to VMGA III transport medium which preserved the viability of the bacteria.

\section{Isolation and identification of dental plaque associated bacteria}

Pathogenic bacteria isolated from the dental plaque were cultured on the Mitis- Salivarus agar (MSA) medium for the isolation of Streptococcus mutans. MRS agar as a medium was used for the isolation of Lactobacillus sp. and MSA agar medium for the isolation of Staphylococcus species. These all plates were incubated for overnight at $37^{\circ} \mathrm{C}$. Colony morphology of the bacteria on the plates was observed and Gram staining was performed. All the bacterial isolates were identified using morphological, microscopic and biochemical tests following the standard procedures described by Cowan and Steel (1974). Biochemical tests were conducted to identify the Staphylococcus, Streptococcus and Lactobacillus species. These testes included Nitrate reduction, Motility, Oxidase, Urease, Catalase, Citrate and Methyl Red.

\section{Reference Strains used in the study}

Three strains of bacteria namely Streptococcus mutans (MTCC 497) Staphylococcus coccus aureus (MTCC 3160) and Lactobacillus acidophilus (MTCC 10307) were procured from Microbial Type Culture Collection and Gene Bank (Institute of Microbial Technology) Chandigarh, India. These organisms were subcultured and used as reference strains.

\section{Collection of plant material}

The bark of the plant Salix alba was collected from Kashmir, India. It was identified and authenticated in the Department of Botany Annamalai University. The bark was washed with sterile water and dried at room temperature. 


\section{Synthesis of Silver nanoparticles from Salix alba} bark extract

Plant material and preparation of extract

The bark of young branches of Salix alba used in the study was collected from Tral Jammu and Kashmir and identified in the department of Botany Annamalai University Chidambaram Tamil Nadu. The bark was washed extensively for three times with sterile distilled water, the bark was then sun dried and grounded to make a fine powder. Further 4 grams of powder were taken into $250 \mathrm{ml}$ beaker added with $100 \mathrm{ml}$ of sterile distilled water and boiled for $10 \mathrm{~min}$ at $100^{\circ} \mathrm{C}$. The whole mixture was filtered through whatman filter paper and plant extract was collect in the beaker and used for further experiment.

\section{Chemicals}

Silver nitrate was purchased from SigmaAldrich, Bangalore, India. The bacteriological media were purchased from HiMedia Laboratories, India. All media and solutions were prepared in doubledistilled Milli $Q$ water.

\section{Biosynthesis of silver nanoparticles}

The procedure for the preparation of the silver nanoparticles has been adopted from ${ }^{8-9}$ with slight modifications. $1 \mathrm{mM}$ AgNO3 (silver nitrate) solution was prepared and stored in amber colour bottle. $10 \mathrm{ml}$ of whole plant extract was taken in beaker separately and $50 \mathrm{ml}$ of $1 \mathrm{mM} \mathrm{AgNO} 3$ solution was added to the beaker drop wise with constant stirring at $50-60^{\circ} \mathrm{C}$ and colour change was observed from yellow to dark brown indicates the formation of nanoparticles and absorbance was measured by using UV-vis spectrophotometer.

\section{Characterization techniques UV-Vis Spectroscopy}

The reduction of silver ions in to silver nanoparticles was confirmed by UV-Visible spectroscopy. $1 \mathrm{ml}$ sample of silver nanoparticle solution was taken in a quartz cuvette and observed for wavelength scanning between 300-700 nm. PerkinElmer Lambda 950 UV/Vis spectrometer was used for UV Visible spectroscopy.
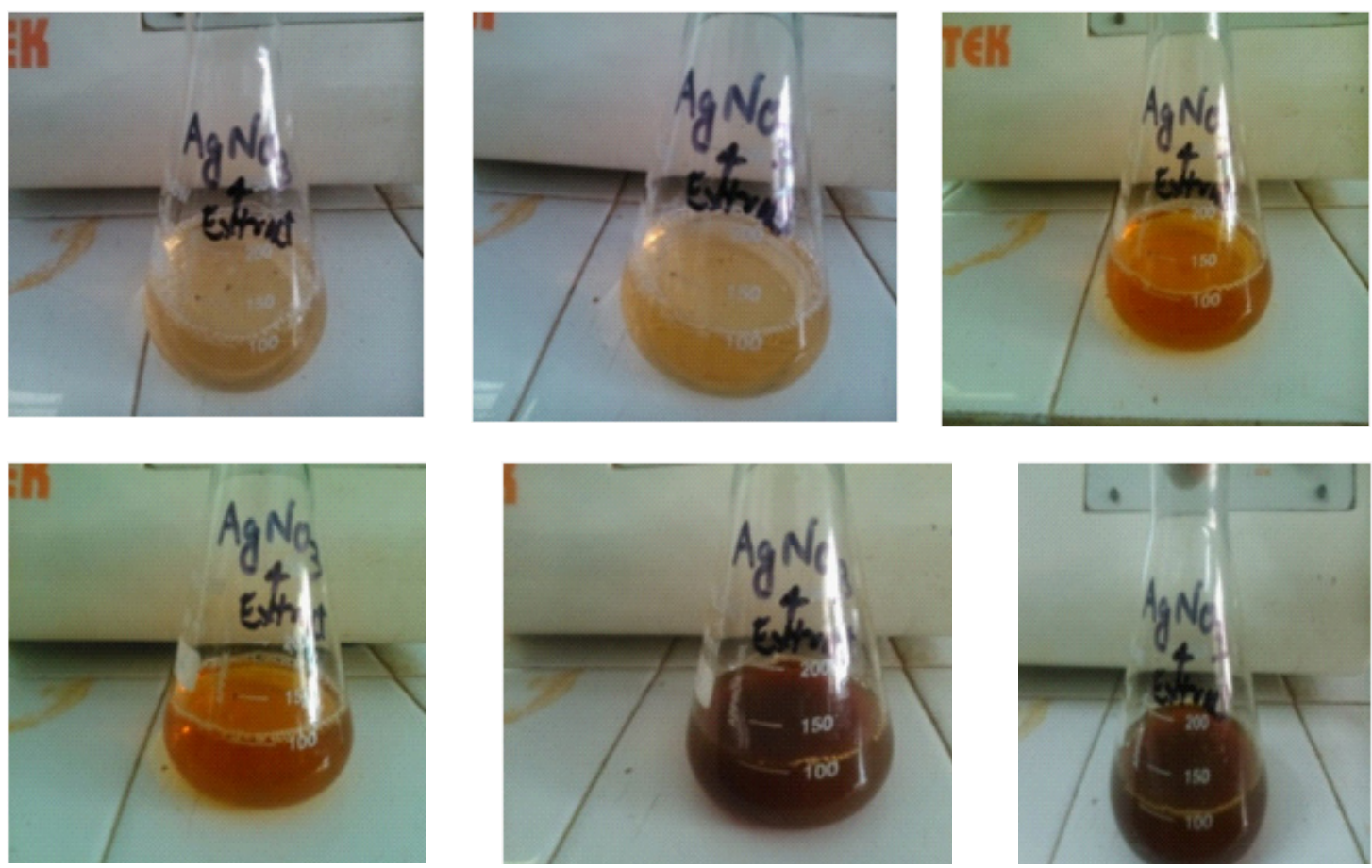

Fig. 1: Colour change observed in the sample at different time intervals.4.8 upon addition of $\mathrm{AgNO}_{3}$ 


\section{Zeta $(\zeta)$ Potential Analysis}

The zeta $(\zeta)$ potential analysis of the silver nanoparticles was carried out to find out the stability of silver nanoparticles. The prepared sample of nanoparticles was dispersed in deionized water followed by ultra-sonication for 5 minutes. After sonication solution was filtered and centrifuged for $15 \mathrm{~min}$. at $25^{\circ} \mathrm{C}$ with $5000 \mathrm{rpm}$ and the supernatant was collected. The supernatant was diluted for 4 to 5 times and then the particle distribution in liquid was studied in a computer controlled particle size analyzer (ZETA sizer Nanoseries, Malvern instrument Nano $\mathrm{Zs})$.

\section{Atomic Force Microscopy (AFM)}

Atomic force microscopy (AFM) is an advanced characterization technique to determine the shape, size and dispersion of the silver nanoparticles. In order to characterize the silver nanoparticles, the sample was prepared by sonication at room temperature for about 15 minutes in the ultra sonicator. Then the sample solution was dried as a thin on a glass slide and prepare a thin film and subject to AFM analysis.

\section{Transmission Electron Microscope (TEM)}

Transmission Electron Microscope (TEM) offers the highest resolution which can be achieved. This method is used to observe the particle size of a material in nano-dimension and study the crystal structure meticulously.

In the high resolution transmission electron microscope (HR-TEM) a thin sample is irradiated with a sharp high energy electron beam (usually in the range 100-200 KeV). The important applications of TEM include the observation of nanostructures and morphology, study of the crystalline and nanocrystalline structure, TEM analysis was done to know the particle size and other crystalline information in this study.

Sample for TEM analysis was prepared by drop- coating Ag nanoparticles onto carbon-coated

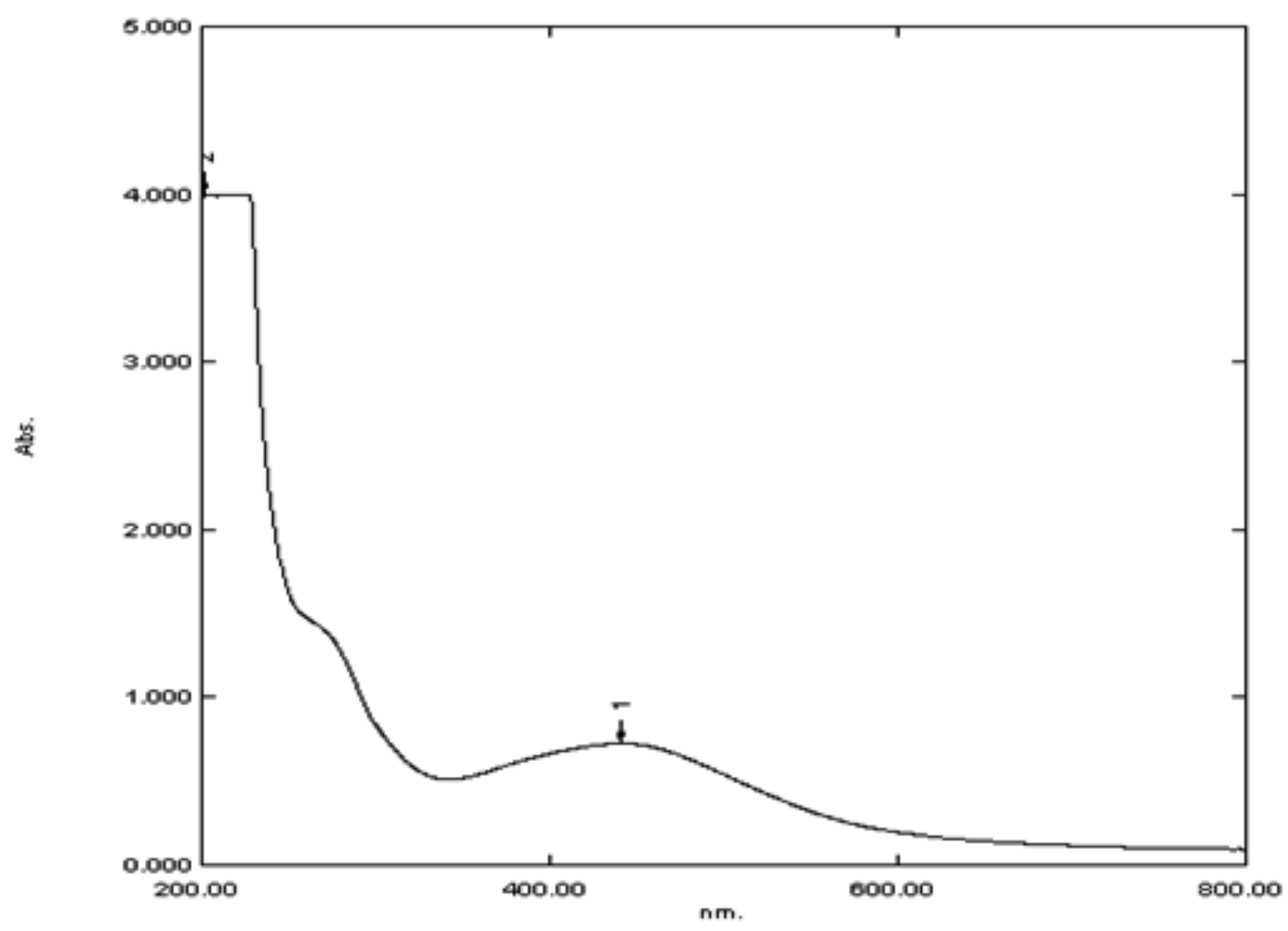

Fig. 2: UV-Vis spectroscopy of the Salix alba bark extract added with silver nitrate 
copper TEM grids. The film on the TEM grids were allowed to stand for 2 mins, following which the extra solution was removed using a blotting paper and the grid was allowed for drying prior to the measurement. TEM micrographs of the sample were taken using the JEOL JSM 100CX TEM instrument operated at an accelerating voltage of $200 \mathrm{kv}$.

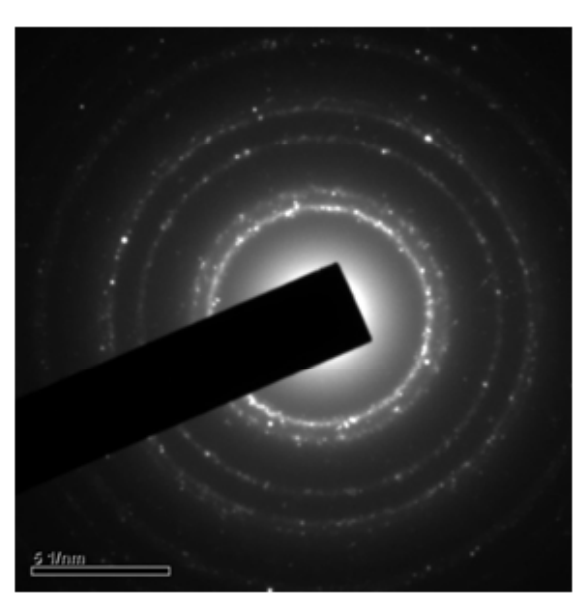

\section{Determination of anti-microbial activity of AgNPs} by disc diffusion method

The petri plates were prepared with 20 $\mathrm{mL}$ media of sterile Mueller Hinton Agar (MHA) and the test organism cultures like lactobacillus $\mathrm{sp}$., streptococcus sp. and staphylococcus sp. isolated from the dental plaque were swabbed on the solidified media and keep for $10 \mathrm{~min}$.. Each

Fig. 3: HR-TEM Transmission observation of nanoparticles

\section{Zeta Potential Distribution}

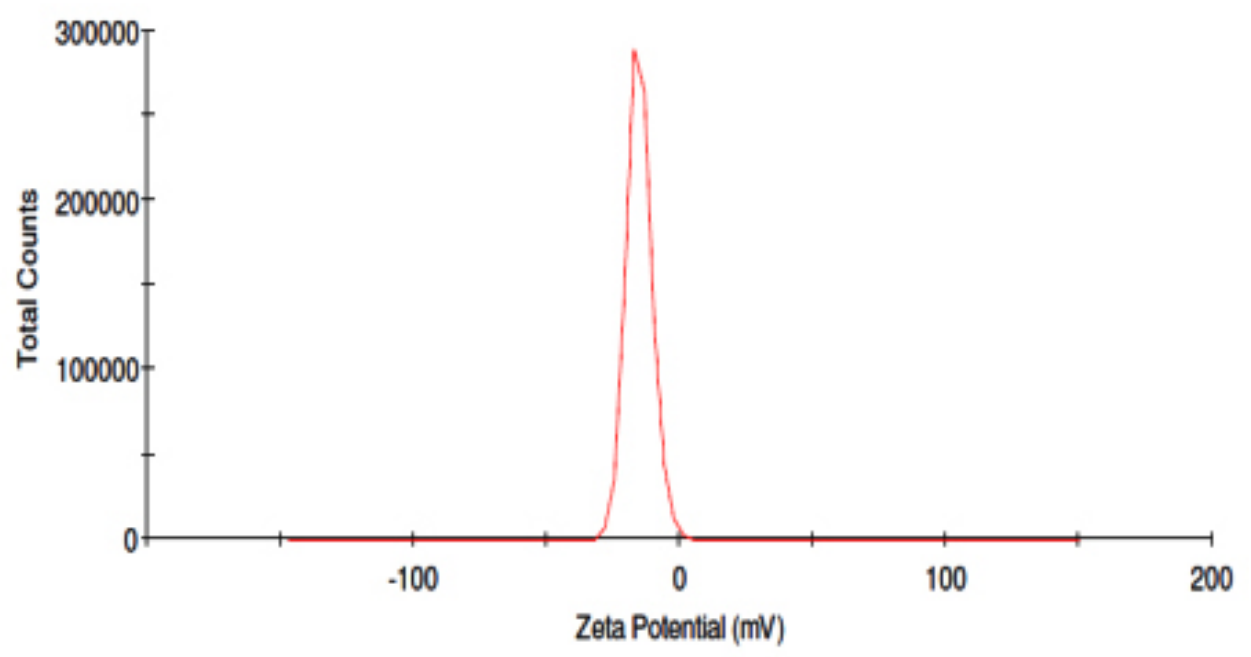

\section{Record 17:1-zeta 1}

Fig. 4: The Zeta potential of the biosynthesized Silver nanoparticles from Salix alba 
disc was impregnated with the concentrations of $40 \mu \mathrm{g}$ of biosynthesized silver nanoparticles which were placed on the surface of the solidified agar medium ${ }^{10}$. These nanoparticles were compared with different antibiotics like amoxicillin, tetracycline and ciprofloxacin. The plates were incubated for 24 hours at $37^{\circ} \mathrm{C}$ for bacterial growth. Zones of inhibition were recorded in millimeters $(\mathrm{mM})$ and the experiment was repeated thrice. All the data were statistically analyzed.

\section{RESULT AND DISCUSSION}

In this proposed study Salix alba was first time used for the biosynthesis of silver nanoparticles. The aqeous extract of plant salix alba when challenged with silver nitrate $(1 \mathrm{mM})$ which results in the quick change of the color in to dark brown which was s noted by visual observation (Fig. 1) with the increasing period of incubation.due to surface
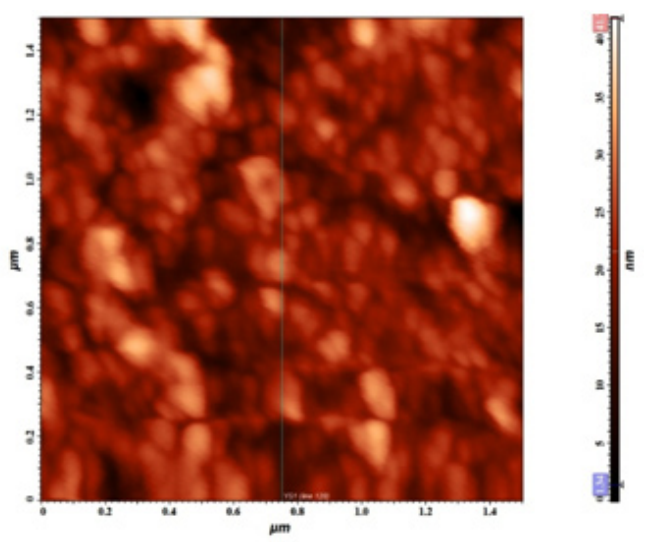

Fig. 5: Atomic Force Microscopy images of biosynthesized nanoparticles from Salix alba plasmon resonance (SPR) of silver nanoparticles The color change at different time intervals can be observed as shown Fig 1(Mulvaney 1996) ${ }^{11}$.

The reduction of silver ion in to silver nanoparticles was analyzed by spectral data obtained by using a UV-Vis spectrophotometer analysis which is the common technique for qualitative analysis of nanoparticles 12]. It showed an absorbance peak around $440 \mathrm{~nm}$ (Fig.2) which confirms the specific for silver nanoparticles ${ }^{11,12,13}$.

TEM analysis showed that the average mean size of silver nanoparticles was between 29-35 $\mathrm{nm}$ and the tiny particles were seemed to be spherical in morphology as shown in the following images (Fig. 3). The images also showed the existence of nano crystalline structure in the particles. The selected area electron diffraction (SAED) analysis showed those continuous ring patterns which originate from polycrystalline state are by the more crystallites attached to the surface of the single particles. Bright ring pattern showed the high density of crystallites in the material.

The zeta potential test was used to measure the electrophoretic mobility and charge of each nanoparticle sample when the zeta potential value is high indicates a high electric charge on the surface of the NPs which describes strong repellent forces among the particles, which prevent aggregation and lead to stabilizing the NPs in the medium. At natural provision $(\mathrm{pH}=7.2)$, the values for the zeta potential was -15.1 , width $(\mathrm{mV})$ with $100 \%$ intensity. This result revaluated the synthesized silver nanoparticles that were stable due to the electrostatic repulsion without adding a different physical or chemical capping

Table 1: Antibacterial Activity of silver nanoparticles and antibiotics, Zone of inhibition measured in $\mathrm{mM}$

\begin{tabular}{lccccccc}
\hline Pathogens & AgNPs & Amox & $\begin{array}{c}\text { Amox } \\
\text { +AgNPs }\end{array}$ & Tet & $\begin{array}{c}\text { Tet } \\
\text { +NPs }\end{array}$ & Cip & $\begin{array}{c}\text { Cip } \\
\text { + AgNPs }\end{array}$ \\
\hline Staphylococcus sp & $15 \pm 0.34$ & $11 \pm 0.11$ & $17 \pm 0.14$ & $15 \pm 1.10$ & $21 \pm 0.21$ & $22 \pm 0.32$ & $29 \pm 0.83$ \\
Streptococcus sp. & $18 \pm 0.69$ & $10 \pm 0.74$ & $14 \pm 0.39$ & $13 \pm 0.93$ & $18 \pm 0.23$ & $20 \pm 0.21$ & $27 \pm 0.76$ \\
Lactobacillus sp. & $19 \pm 0.45$ & $12 \pm 0.33$ & $16 \pm 0.22$ & $19 \pm 0.32$ & $26 \pm 0.16$ & $24 \pm 0.17$ & $31 \pm 0.21$
\end{tabular}

Where Amox- Amoxicillin, Tet- Tetracycline, Cip- Ciprofloxacin and AgNPs - Silver nanoparticles, \pm Average standard deviation 
agent. This is very important for use for therapeutic propose (Fig. 4).

\section{Salix alba}

Atomic Force Microscopy an advanced technique was used to characterize the synthesized nanoparticles. AFM is used to identify the size, shape and dispersion of the nanoparticles AFM analysis showed that the particle size is between 40-50nm (Fig. 6) which are spherical in shape ${ }^{14}$.

These silver nanoparticles were evaluated for its antibacterial effect against bacteria isolated from dental plaque samples obtained from the Raja Muthiah Dental College like Streptococcus sp., Lactobacillus sp. and Staphylococcus species. Antibacterial analysis was carried out by using disc diffusion method. The bacterial strains were gown on the Mueller Hinton Agar (MHA) media by using sterile cotton to swab on the media. Each disc was loaded with $40 \mu \mathrm{g}$ of silver nanoparticles which showed good antibacterial activity with maximum zone of inhibition was $19 \mathrm{mM}$ for lactobacillus sp. followed by Streptococcus and staphylococcus $\mathrm{sp}$. with $18 \mathrm{mM}$ and $15 \mathrm{mM}$ respectively.. These nanoparticles were compared with different antibiotic like Amoxicillin, tetracycline and ciprofloxacin respectively. For checking the synergistic effect each disc is loaded with half of the nanoparticle means $20 \mu \mathrm{g}$ per antibiotic disc. The highest zone of inhibition was shown by lactobacilli species was $31 \mathrm{mM}$ in combination with ciprofloxacin and nanoparticles followed by tetracycline of lactobacillus was $19 \mathrm{mM}$ followed by staphylococcus was $17 \mathrm{mM}$ in amoxicillin ${ }^{15}$ These antibiotics showed very good antibacterial activity alone but there antibacterial activity enhances quite significantly as shown in Table no.1.Fig 6 showed the graphical representation of antibacterial effect silver nanoparticles with various antibiotics. Each experiment was repeated three times and average value has been calculated by using standard deviation. These isolated strains were compare with reference strains of Streptococcus mutans (MTCC 497) Staphylococcus coccus aureus (MTCC 3160) and Lactobacillus acidophilus (MTCC 10307) were procured from Microbial Type Culture Collection and Gene Bank (Institute of Microbial Technology). Hence this may open a new way to control the infection by reducing the antibiotic dosage and enhance the efficacy of antibiotics to fight the bacterial infections in a much more powerful manner to eradicate the oral infection which in turn also decrease the infection to disseminate other parts of body.

\section{Graphical representation}

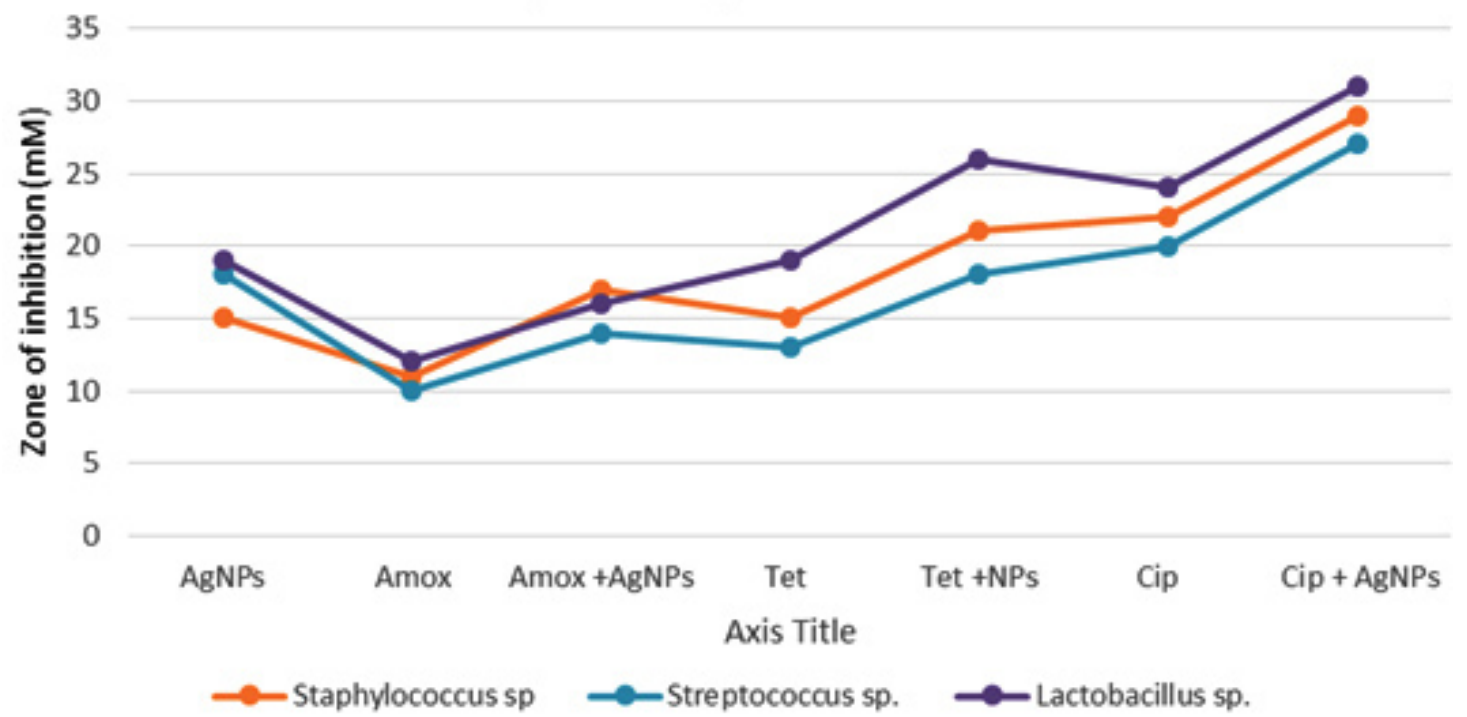

Fig. 6: Graphical representation of antibacterial activity of silver nanoparticles along with different antibiotics 
Salix alba is first time employed for the biosynthesis of silver nanoparticles which showed the rapid synthesis of nanoparticle as well as showed good antibacterial effect against dental carries bacteria in a powerful manner.

\section{CONCLUSION}

Salix alba first time used for the biosynthesis of silver nanoparticles and acts as a good candidate for the reduction of silver ions into silver nanoparticles. Silver nanoparticles synthesized from the Salix alba showed good antibacterial activity lone against the isolated bacterial pathogens from dental plaque and also enhances the efficacy of antibiotics quite remarkably. . Hence could become strong antibacterial agent for the oral infections and also antibiotics which can decrease the antibiotics dosage with improved antibiotics efficacy, but before using as a strong bacterial material for the oral infections needs animal trial to check its toxicity.

\section{ACKNOWLEDGMENTS}

The authors would like to acknowledge the Department of Pharmacy, University of Kuala Lumpur, Royal college of medicine, Ipoh, Malaysia and Department of Microbiology Annamali University, Chidambaram Tamil Nadu, India for providing the necessary facilities to carry out this research.

\section{REFERENCES}

1. Rai M. K.; Deshmukh S. D.; Ingle A.; and A.K. Gade, . J. Appl. Microbiol 2012.112(5), 841852

2. Shrivastava.S.; Bera T.; Roy A.; Singh G.; Ramachandrarao Dash P.. Journal of Nanotechnology, 2007 18., 225103-225112.

3. Roh Y, Bai J, Lauf RJ, Mcmillan AD, Phelps TJ, Rawn CJ, Solid State Commun, 2001.. 118, 529-34.

4. Sastry M.; Ahmad A.; Islam Khan M.; Kumar R. Current Science, 2003, 85,162-70..

5. Ankamwar B.; Damle C.; Ahmad A.; and Sastry M., Journal of NanoscienceNanotechnology, 2005,5, . 1665-71

6. Priya Banerjee.; Mantosh Satapathy.; Aniruddha Mukhopahayay and Papita Das . Bioresources and Bioprocessing, 2014, 1, 2- 10.

7. Bansal V.; Rautaray D.; Ahmad D. and Sastry M. Journal of Material Chemistry 2004., 14. $3303-3305$.
8. Gardea Torresdey J.L.; Parson J.G.; Gomez E.; Peralta Videa J.; Troiani H.E. and Santiago P. Journal of American Chemical Society. 2002. 2, 397-401.

9. Dasaratrao Sawle B.; Salimath B.; Deshpande R.,;Bedre M.D.; Prabhakar B.K. and Venkataraman A. Journal of Science and Technology of Advanced Materials, 2008. 9. 6.

10. Bauer A.W; Kirby M, Sherris J.C.; Truck M. Am J Clin Pathol. 1996;. 45:493-6.

11. Mulvaney P.,Langmuir. 1996 12. 788-800.

12. Chudasam B.; Vala A, Andhariya N., Mehta R., and Upadhyay., Journal of Nano Res.. 2009.2, 955- 965.

13. Vahabi K.; Mansoori G.A.; Karimi .S, . In science J. 2011., 1,65-79.

14. Subramaniam M, Alikunhi NM, Kandaasamy K... Ad Sci Lett. 2010 3. 428-33. 27.

15. Nanda $A$ and Majeed S., Int j pharm pharm sci, 2014., 6,(2), 609-612. 